\title{
IMPACTO DE LA MORTALIDAD FETAL SOBRE LA PREMATUREZ EN ARGENTINA: ESTUDIO POBLACIONAL
}

\author{
IMPACT OF FETAL MORTALITY ON PREMATURITY IN ARGENTINA: A POPULATION BASED STUDY
}

Carlos Grandi, ${ }^{1,2}$, Ligia Nascente ${ }^{3}$, Viviane C.Cardoso ${ }^{4}$

\section{Resumen:}

Antecedentes: Se ha observado que el aumento de nacimientos prematuros fue acompañado por una disminución en la mortalidad fetal.

Objetivo: Evaluar la relación entre la mortalidad fetal y la prematurez en Argentina.

Material y Métodos: Diseño ecológico; la población incluyó a todos los nacidos vivos y muertos de Argentina entre 2003 y 2013.

Variables Resultado: tasa de mortalidad fetal total (TMFT), tasa de mortalidad fetal por peso, razón de mortalidad fetal, tasa de mortalidad neonatal, tasa de mortalidad perinatal, tendencias de las tasas de mortalidad fetal específicas por edad gestacional (EG), y Prematurez ( $P P,<37^{+0}$ semanas). Variables independientes: edad ( $<19$ y $>35$ años), educación $<8$ años, con pareja, paridad 1 y $>4$, embarazo múltiple y tasa de natalidad.

Análisis estadístico: análisis de regresión lineal ajustado para covariados.

Resultados: La TMFT mostró una reducción anual del 0,18 por mil y disminución con el aumento de la EG, siendo la mayor reducción en menores a $28^{+0}$ semanas $(68 \%)$, mientras que el parto prematuro aumentó $0,07 \%$ por año, siendo el mayor incremento $(0,75 \%)$ entre la $32^{+0}$ y $36^{+6}$ semanas. La regresión lineal mostró un $\mathrm{R}^{2}$ ajustado de $0,76(\mathrm{p}=0,010)$ y la mayor relación inversa entre la TMFT y el PP se observó en prematuros entre $32^{+0}$ a $36^{+6}$ semanas $\left(R^{2}=0,98, p=<0,001\right)$.

Conclusiones: La reducción de la mortalidad fetal se asoció con el incremento observado de los nacimientos vivos prematuros en la Argentina.

Palabras clave: mortalidad fetal; prematuro; mortalidad neonatal; tendencias; diseño ecológico.

\section{Abstract:}

Background: It has been observed that the increase in preterm births was accompanied by a decrease in fetal mortality.

Objective: To evaluate the relationship between fetal mortality and prematurity in Argentina.

Methods: Ecological design. The population included all live births and fetal demise in Argentina between 2003 and 2013. Outcomes: total fetal mortality rate (TFMR), fetal mortality rate by birth weight, fetal mortality rate, neonatal mortality rate, perinatal mortality rate, trends in fetal mortality rates specific for gestational age (GA), and prematurity ( $<37+0$ weeks). Independent variables: age ( $<19$ and $>35$ years), education $<8$ years, with partner, parity 1 and $>4$, multiple pregnancy and birth rate. Statistical analysis: linear regression analysis adjusted for covariates.

Results: TFMR showed an annual reduction of 0.18 per thousand births that decreased with the increase of $\mathrm{GA}$, being the largest reduction below $28^{+0}$ weeks (68\%), while prematurity increased $0.07 \%$ per year, being the largest increase $(0.75 \%)$ between $32^{+0}$ and $36^{+6}$ weeks. Linear regression model showed an inverse relationship between TFMR and prematurity (adjusted $R^{2} 0.76, p=0.010$ ), being the highest between $32^{+0}$ and $36^{+6}$ weeks (adjusted $R^{2}=0.98, p<0.001$ ).

Conclusions: The reduction in fetal mortality was associated with the observed increase in preterm births in Argentina.

Keywords: mortality; premature; neonatal mortality; trends; ecological design.

\footnotetext{
${ }^{1}$ Doctor en Medicina. Especialista en Pediatría y Neonatología. Ex Investigador independiente, Consejo de Investigación en Salud, Buenos Aires, Argentina.

${ }^{2}$ Email de contacto: cgrandi@intramed.net

${ }^{3}$ Médica. Residente de Pediatría. Facultad de Medicina de Ribeirão Preto, Universidad de San Pablo, Brasil.

${ }^{4}$ Doctora en Medicina. Profesora. Departamento de Puericultura y Pediatría. Facultad de Medicina de Ribeirão Preto, Universidad de San Pablo, Brasil.
} 


\section{Introducción}

El número de niños nacidos muertos anuales se mantiene sin cambios desde 2011 y es inaceptablemente alto; se estima que en América Latina en 2015 la tasa de mortalidad fetal (>28 semanas) fue de 8,7 por 1000 nacidos vivos ${ }^{(1)}$. La falla en incluir de forma consistente indicadores de muerte fetal en las estadísticas vitales nacionales e iniciativas para después de 2015 muestra que los niños nacidos muertos están ocultos para la agenda mundial ${ }^{(2)}$.

Los avances en medicina fetal y cuidados intensivos neonatales están siendo disponibles rutinariamente, por lo que en los recién nacidos (RN) que no crecen bien en el útero el parto puede anticiparse, reduciendo las muertes fetales, especialmente las tardías, pero a expensas de un aumento de las tasas de nacimientos prematuros (RN vivos $<37^{+0}$ semanas) ${ }^{(3)}$.

Esto es consistente con la hipótesis de que la interrupción de la gestación en los embarazos de alto riesgo puede aumentar la tasa de supervivencia de los fetos y por lo tanto reducir la mortalidad perinatal ${ }^{(4,5)}$. Esta hipótesis está apoyada por la similitud entre los factores de riesgo de nacimientos prematuros y las muertes fetales y neonatales ${ }^{(6)}$.

A pesar de tener información de alta calidad Argentina no dispone de información poblacional sobre las tendencias del parto prematuro y su relación con la mortalidad fetal. Por tal motivo el presente estudio tuvo como objetivo evaluar la relación en el tiempo entre los nacidos vivos prematuros y las muertes fetales y neonatales en Argentina según ciertos indicadores.

Nuestra hipótesis es que la reducción de la mortalidad fetal se asocia con el incremento observado de la prematurez en la Argentina.

\section{Métodos}

\section{Diseño del estudio y población}

Éste es un estudio de diseño ecológico, usando el país como unidad de análisis. La población de estudio incluyó a todos los nacidos vivos y muertos en Argentina durante el período 20032013. Los datos, de acceso libre, fueron obtenidos de datos agregados de las Estadísticas Vitales de la Dirección de Estadística e Información de Salud (DEIS) del Ministerio de Salud ${ }^{(7)}$.

\section{Variables Resultado}

La OMS recomienda que todas las muertes fetales que pesan $500 \mathrm{~g}$ o más se incluyan en las estadísticas vitales ${ }^{(8)}$. Si no se cuenta con el peso al nacer (PN), la OMS recomienda el uso de la edad gestacional (EG) correspondiente $\left(22^{+0}\right.$ semanas o más), criterio adoptado por la DIES.

Se calcularon las siguientes variables resultado: a) tasa de mortalidad fetal total (TMFT): es el cociente entre las defunciones fetales totales y el total de nacidos (vivos y muertos), por mil; b) tasa de mortalidad fetal por peso: es el cociente entre las defunciones fetales de 1000 gramos y más y el total de nacidos (vivos y muertos) de $1000 \mathrm{~g}$ y más, por mil; c) razón de mortalidad fetal (RMF): es el cociente entre las defunciones fetales totales y el total de nacidos vivos, por mil; d) tasa de mortalidad neonatal (TMN): Nro. de muertes de niños de menos de 28 días de vida/ Nro. de nacidos vivos, por mil; e) tasa de mortalidad perinatal: es el cociente entre la suma de las defunciones fetales tardías $\left(22^{+0}\right.$ semanas y más) más el Nro. de defunciones de RN menores de 7 días y la suma de las defunciones fetales tardías y Nro. de nacidos vivos, por mil; f) tendencias de las tasas de mortalidad fetal específicas (TMFE) por EG (entre 2011 y 2013 por ser los únicos años con información del número de nacidos vivos por $E G$, ya sean nacimientos únicos o múltiples) y g) Prematurez se definió como una EG menor a $37^{+0}$ semanas.

\section{Variables independientes}

La DIES emplea la fecha de la última menstruación (FUM) para estimar la edad gestacional y hasta 2010 sólo informó la EG de los $\mathrm{RN}$ vivos categorizándola en Pretérmino: menos de $37^{+0}$ semanas (menos de 259 días), a Término: de $37^{+0}$ a $41^{+6}$ semanas (259 a 293 días) y Postérmino: $42^{+0}$ semanas o más (294 días o más). A partir de 2011 la distribución de los prematuros vivos por EG fue: $<28^{+0}$ semanas (extremadamente prematuros); $28^{+0}$ a $31^{+6}$ semanas (muy prematuros) y entre $32^{+0}$ y $36^{+6}$ semanas (prematuros leves/moderados).

$\mathrm{Se}$ registraron las siguientes características maternas: edad ( $<19$ y $>35$ años), educación $<8$ años, con pareja, paridad 1 y $>4$ y embarazo múltiple. Además, se registró la tasa de natalidad.

\section{Análisis estadístico}

La descripción de las variables se hizo a partir de la determinación de las medidas de tendencia central (media, mediana y proporciones) y de dispersión (desviación estándar [DS], intervalo intercuartil [IIC] e intervalo de confianza del 95\% [IC 95\%]).

Las tendencias anuales de las características maternas se analizaron mediante la prueba de chi2 para tendencias de proporciones y las variaciones anuales de las tasas de natalidad, 
mortalidad, la relación entre la tasa de mortalidad fetal y prematurez y las tendencias en el tiempo para todas las variables se calcularon mediante el análisis de regresión lineal.

Se ajustaron tres modelos de regresión múltiple para analizar las asociaciones entre parto prematuro con la mortalidad fetal total, la mortalidad neonatal y la mortalidad perinatal, ajustadas para la proporción de madres de 35 años de edad o más, multíparas y embarazo múltiple.

Por último, se calculó una matriz de correlación de Pearson entre las tendencias de las características maternas y la mortalidad fetal con la prematurez.

El nivel de significación estadística se fijó en 0,05 y los análisis se realizarán utilizando el programa Stata versión 13.0 (College Station, Texas, EE.UU.).

\section{Aspectos éticos}

El estudio fue aprobado por el Consejo de Evaluación Ética de Investigación en Salud (CoEIS), Córdoba, Argentina.

\section{Resultados}

La proporción de datos incompletos de la edad gestacional fue 2,6\% para el total de los nacidos vivos y $3,4 \%$ para las muertes fetales; esto fue menor del $5 \%$ para todas las otras variables analizadas. La muestra final estuvo integrada por 8.042.611 RN vivos y 57.472 fetos muertos (total 8.100 .083 registros).

La Tabla 1 muestra la distribución de las características maternas por año; se observó una declinación anual del $0,22 \%$ de la tasa de natalidad, $0,33 \%$ para educación primaria incompleta y $0,57 \%$ para multíparas, mientras que se incrementaron $0,17 \%$ el embarazo adolescente, $0,14 \%$ las madres $\geq 35$ años y $0,06 \%$ el embarazo múltiple, todas estadísticamente significativas.

Entre 2003 y 2013, 5,7\% (IC 95\% 5,6 - 5,8) del total los RN pretérminos (nacidos vivos más muertos) fueron muertes fetales (39.612 / 685.902) y, de acuerdo a la distribución por subgrupos de $E G$, entre 22 y 27 semanas $34,6 \%$, entre 28 y 31 semanas $24,9 \%$ y entre 32 y 36 semanas $40,4 \%$.

Tabla 1: Tasa de Natalidad y Características maternas (Argentina 2003-2013)

\begin{tabular}{|c|c|c|c|c|c|c|c|c|}
\hline AÑO & TASA DE NATALIDAD $\% 0$ & Edad $\leq 19$ años $\%$ & Edad $\geq 35$ años $\%$ & EDU < 8 años $\%$ & CON PAREJA \% & Paridad $1 \%$ & Paridad $\geq 4 \%$ & E.MULTIPLE \% \\
\hline 2003 & 18,20 & 13,80 & 13,60 & 9,20 & 84,00 & 36,90 & 20,61 & 1,60 \\
\hline 2004 & 19,30 & 14,50 & 13,40 & 9,50 & 83,50 & 37,20 & 20,20 & 1,69 \\
\hline 2005 & 18,50 & 15,10 & 13,60 & 9,60 & 85,80 & 39,00 & 18,40 & 1,76 \\
\hline 2006 & 17,90 & 15,40 & 13,60 & 9,00 & 86,80 & 40,10 & 17,10 & 1,79 \\
\hline 2007 & 17,80 & 15,70 & 13,90 & 9,50 & 86,90 & 40,40 & 16,70 & 1,74 \\
\hline 2008 & 18,80 & 15,50 & 13,80 & 8,80 & 86,50 & 40,30 & 16,30 & 1,86 \\
\hline 2009 & 18,60 & 15,80 & 14,00 & 8,60 & 86,30 & 40,10 & 16,20 & 1,87 \\
\hline 2010 & 18,70 & 15,50 & 14,20 & 7,80 & 89,30 & 40,00 & 15,80 & 1,71 \\
\hline 2011 & 18,50 & 15,70 & 14,70 & 6,90 & 79,50 & 39,60 & 14,80 & 1,88 \\
\hline 2012 & 17,90 & 15,70 & 15,20 & 6,20 & 85,20 & 40,80 & 14,00 & 1,78 \\
\hline 2013 & 17,90 & 15,70 & 15,40 & 5,50 & 85,30 & 40,90 & 13,40 & 2,01 \\
\hline MEDIA & 18,37 & 15,31 & 14,13 & 8,24 & 85,37 & 39,57 & 16,68 & 1,79 \\
\hline DS & 0,45 & 0,60 & 0,65 & 1,37 & 2,49 & 1,35 & 2,32 & 0,11 \\
\hline MEDIANA & 18,50 & 15,50 & 13,90 & 8,80 & 85,80 & 40,10 & 16,30 & 1,78 \\
\hline 1er CUARTIL & 17,90 & 15,25 & 13,60 & 7,35 & 84,60 & 39,30 & 15,30 & 1,73 \\
\hline 3er CUARTIL & 18,65 & 15,70 & 14,45 & 9,35 & 86,65 & 40,35 & 17,75 & 1,87 \\
\hline
\end{tabular}

EDU: educación

E.MULTIPLE: embarazo múltiple

El total de nacidos vivos prematuros fue 52.028 en 2003 y 64.389 en 2013, lo que representa un incremento en la tasa de prematurez de 0,07\% por año, pasando de $7,9 \%$ en 2003 a $8,6 \%$ en 2013 ( $p<0,001$, Figura 1). Entre 2011 y 2013 ( $n=$ 190.319) la distribución de la EG en nacidos vivos prematuros fue: $<28$ semanas, 3,8\%; 28 a 31 semanas, $8,9 \%$ y entre 32 y 36 semanas, $87,2 \%$. En el mismo período los nacimientos prematuros aumentaron $0,42 \%$ en el grupo de prematuros extremos y $0,75 \%$ en prematuros moderados, mientras que disminuyó $1,1 \%$ en los muy prematuros.

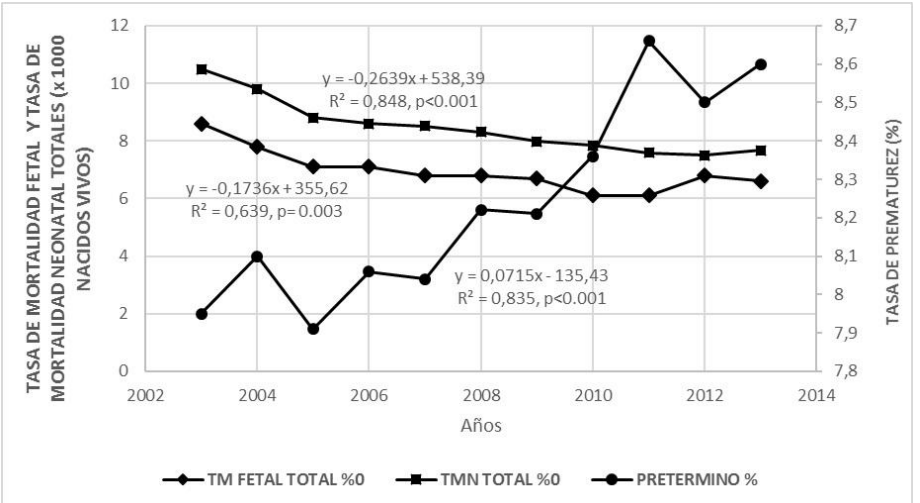

Figura 1. Evolución de la Tasa de Mortalidad Fetal Total, Tasa de Mortalidad Neonatal y proporción de RN Prematuros vivos (Argentina 2003-2013) TM: Tasa de Mortalidad 
El número total de defunciones fetales fue de 5.998 en 2003 y 5.017 en 2013; 70,2\% fueron predominantemente prematuras.

La TMFT mostró una reducción anual del 0,18 por mil, pasando de 8,6 en 2003 a 6,6 por mil en 2013 (Figura 1, $\mathrm{p}=0,003$ ). La tasa de mortalidad fetal por peso pasó de 6,4 a 4,7 por mil (promedio anual 0,27 , $\mathrm{p}=0,030)$, mientras que la $R M F$ disminuyo 0,19 anual $(8,7$ a 6,6 por mil, $p=0,003)$, presentando una mayor reducción en los embarazos de pretermino (beta $\left.=-0,94 ; R^{2}=0,90, p<0,001\right)$ que en los de término (beta $=-0,89 ; R^{2}=0,79, p<0,001$ ) y postérmino (beta $=-0,86 ; R^{2}=0,75, p<0,001$ ).

La TMFE disminuyó con el aumento de la EG; en preterminos presentó una reducción anual del 2,1 por mil $(p=0,616)$ mientras que en $R N$ a término fue del 0,07 por mil $(p<0,001)$ y en postérminos 0,04 por mil $(p<0,001)$.

La reducción anual promedio de la tasa de mortalidad neonatal fue un 0,25 por mil (precoz 0,22), de 10,5 a 7,6 muertes por mil (Fig.1), siendo para la mortalidad perinatal de 0,40 por mil $(16,40$ a 11,90$)$; tendencias estadísticamente significativas.

El modelo de regresión lineal mostró que un descenso de 1 por mil de la mortalidad fetal total correspondió a un aumento de $1,86 \%$ de la tasa de prematurez $\left(R^{2}=0,45, p=0,023\right)$ (Figura 2). El ajuste para covariados maternos mostró un mayor valor explicativo $\left(R^{2}\right.$ ajustado $\left.=0,76, p=0,010\right)$. De acuerdo a la $E G(2011-2013)$ la mayor relación inversa entre la mortalidad fetal y la tasa de parto prematuro (PP) se observó en prematuros entre $32^{+0}$ a $36^{+6}$ semanas $\left(R^{2}=0,98, p=<0,001\right)$, debido probablemente a que la mayoría de los PP ocurren en este intervalo.

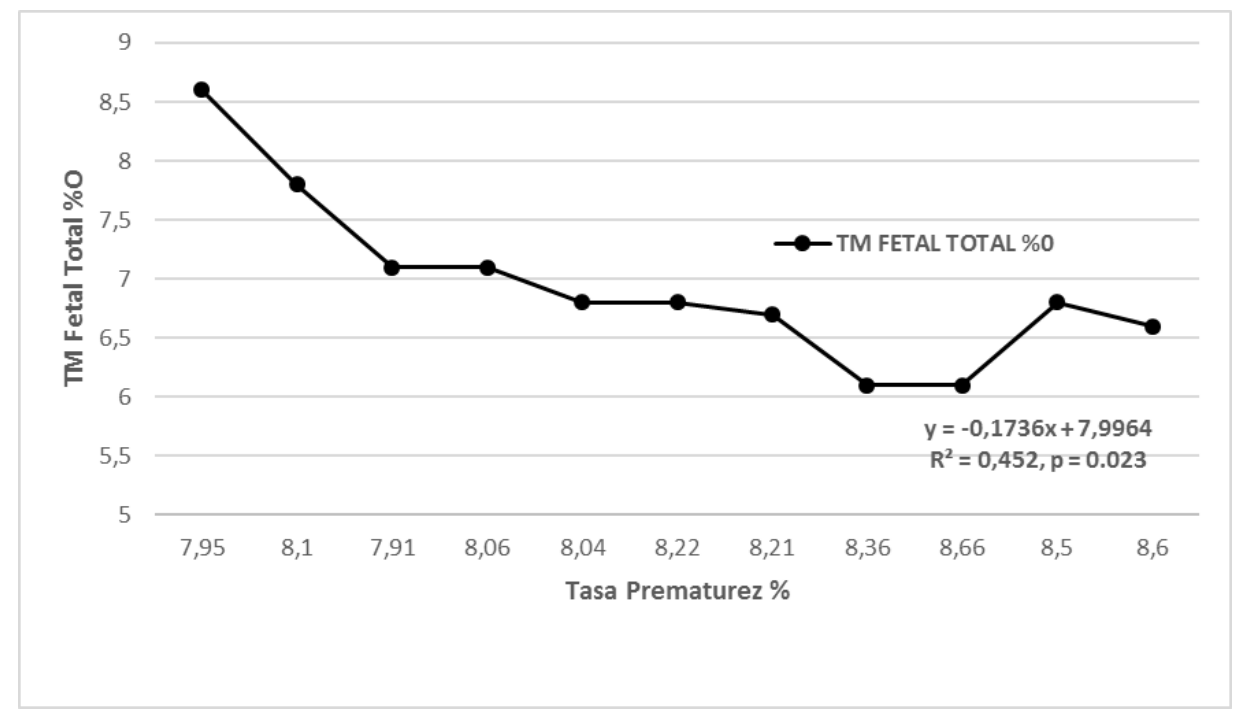

Figura 2: Relación entre la Tasa de Mortalidad Fetal Total y la Tasa de Pretéminos (Argentina 2003-2013) TM: Tasa de Mortalidad

Se observó una asociación más fuerte entre los nacimientos prematuros y las tasas de mortalidad neonatal; un aumento del 1\% en la tasa de nacimientos prematuros se asoció con un descenso del 2,8 por mil en las muertes neonatales $(p=0,006)$. La asociación se hizo más débil después del ajuste: un aumento del $1 \%$ en la tasa de nacimientos prematuros se asoció con una disminución del 0,52 por mil en las tasas de mortalidad neonatal $(p<0,001)$. Finalmente, un aumento del $1 \%$ en la tasa de nacimientos prematuros se asoció con un descenso del 4,1 por mil en las muertes perinatales $\left(R^{2}=0,53, p=0,010\right)$; ajustada para covariados la asociación se incrementó $\left(R^{2}\right.$ ajustado $\left.=0,88, p=0,001\right)$.

La Tabla 2 muestra la matriz de correlación entre las tendencias temporales del parto prematuro y varias características. Hubo correlaciones negativas significativas entre la tendencia de los nacimientos prematuros (que aumentaron en el tiempo) y las tendencias de la proporción de mujeres con pocos años de escolaridad y con la razón de mortalidad fetal (ambas disminuyeron durante el período). Por otro lado, hubo correlaciones positivas y significativas entre las tendencias de los nacimientos prematuros y las tendencias de la gestación múltiple y la edad materna de más de 35 años (ambos aumentados en el período). 
Tabla 2: Matriz de correlación entre las tendencias de las características maternas y la razón de mortalidad fetal con la prematurez (Argentina, 2003-2013)

\begin{tabular}{|c|c|c|c|c|c|c|c|}
\hline Variables & $\underset{a}{\text { Prematurez }}$ & $\begin{array}{c}\text { Embarazo } \\
\text { múltiple }\end{array}$ & $\begin{array}{c}\text { Educación } \\
<8 \text { años }\end{array}$ & $\begin{array}{l}\text { Edad } \\
\text { Materna } \\
\leq 19 \\
\text { años }\end{array}$ & $\begin{array}{c}\text { Edad } \\
\text { Materna } \\
\geq 35 \\
\text { años }\end{array}$ & $\begin{array}{c}\text { Con } \\
\text { Pareja }\end{array}$ & $\begin{array}{l}\text { Razón } \\
\text { de MF }\end{array}$ \\
\hline $\begin{array}{c}\text { Embarazo } \\
\text { múltiple }\end{array}$ & $\begin{array}{c}0.657 \\
(p=0.028)\end{array}$ & 1.000 & & & & & \\
\hline $\begin{array}{c}\text { Educación } \\
<8 \text { años }\end{array}$ & $\begin{array}{c}-0.924 \\
(p<0.001)\end{array}$ & $\begin{array}{c}-0.633 \\
(p= \\
0.036)\end{array}$ & 1.000 & & & & \\
\hline $\begin{array}{c}\text { Edad } \\
\text { Materna } \\
\leq 19 \text { años }\end{array}$ & $\begin{array}{c}0.576 \\
(p=0.063)\end{array}$ & $\begin{array}{c}0.731 \\
(p= \\
0.010)\end{array}$ & $\begin{array}{c}-0.488 \\
(p=128)\end{array}$ & 1.000 & & & \\
\hline $\begin{array}{c}\text { Edad } \\
\text { Materna } \\
\geq 35 \text { años }\end{array}$ & $\begin{array}{c}0.885 \\
(p<0.001)\end{array}$ & $\begin{array}{c}0.635 \\
(p= \\
0.035)\end{array}$ & $\begin{array}{c}-0.974 \\
(p<0.001)\end{array}$ & $\begin{array}{c}0.560 \\
(p= \\
0.073)\end{array}$ & 1.000 & & \\
\hline $\begin{array}{l}\text { Con } \\
\text { Pareja }\end{array}$ & $\begin{array}{c}-0.334 \\
(p=0.315)\end{array}$ & $\begin{array}{c}0.124 \\
(p= \\
0.715)\end{array}$ & $\begin{array}{c}0.211 \\
(p=0.532)\end{array}$ & $\begin{array}{c}0.208 \\
(p= \\
0.538)\end{array}$ & $\begin{array}{c}-0.181 \\
(p= \\
0.593)\end{array}$ & 1.000 & \\
\hline $\begin{array}{c}\text { Razón de } \\
\text { MF }\end{array}$ & $\begin{array}{c}-0.755 \\
(p=0.007)\end{array}$ & $\begin{array}{c}-0.566 \\
(p= \\
0.069)\end{array}$ & $\begin{array}{c}0.728 \\
(p=0.011)\end{array}$ & $\begin{array}{c}-0.648 \\
(p= \\
0.031) \\
\end{array}$ & $\begin{array}{c}-0.814 \\
(p= \\
0.002) \\
\end{array}$ & $\begin{array}{c}0.238 \\
(p=0.480)\end{array}$ & 1.000 \\
\hline
\end{tabular}

a Recién nacidos vivos $<37+0$ semanas

MF: Mortalidad Fetal

\section{Discusión}

Existen muy pocos antecedentes en la literatura argentina sobre el impacto de la mortalidad fetal sobre la prematurez. Los presentes resultados son consistentes con la hipótesis de que la reducción de la mortalidad fetal se asoció con el incremento observado de la prematurez en la Argentina.

La disminución de la mortalidad fetal explicó el $45 \%$ del aumento del parto prematuro en la Argentina en la última década y la mayor relación inversa se observó en prematuros entre $32^{+0}$ y $36^{+6}$ semanas, probablemente atribuible a apropiadas intervenciones obstétricas. Estas cifras son superiores a un estudio ecológico ${ }^{(6)}$ donde el coeficiente de determinación fue de $12 \%$, atribuible a que el estudio se restringió a prematuros tardíos.

La mediana (IIC) de la tasa de PP del período de estudio fue $8,2 \% \quad(8,0$ - 8,4), similar a la comunicada para Latinoamérica en 2010 (8,4\%) (9) , la mayoría entre $32^{+0}$ y $36^{+6}$ semanas, lo que refuerza la solidez de los datos y su utilidad.

Las tasas de mortalidad fetal fueron la mitad de las de América Latina y ligeramente superiores a los países más desarrollados ${ }^{(2)}$. Las muertes fetales por edad gestacional mostraron una distribución en forma de $\mathrm{J}$ y ligeramente similar (aunque un poco mayor) a la de los países desarrollados (10). Cuando los países son categorizados por la tasa de mortalidad fetal $(<5$, $5-14,9,15-24,9$, y $\geq 25$ por 1000 nacimientos totales), existen claras correlaciones con la mortalidad materna y neonatal, así como con los indicadores del sistema de salud ${ }^{(11,12)}$.

La contribución del $5,7 \%$ de las muertes fetales al total de nacimientos prematuros entre 2003 y 2013 concuerda con lo observado en países desarrollados (5-10\%), la mayoría muertes fetales anteparto asociadas a restricción del crecimiento fetal (13).

El momento de la muerte fetal hace referencia a si la defunción ocurrió antes del parto (anteparto) o durante el parto (intraparto). Se ha estimado que, en todo el mundo en 2015, alrededor de la mitad de los nacidos muertos se produjeron durante el parto, y se ha propuesto que, en el caso de prematuros, sean contabilizados en las estadísticas de prematurez ${ }^{(14)}$. La proporción de nacidos muertos intraparto oscila entre 10,0\% (rango 5,5-18,4\%) en las regiones desarrolladas y $59,3 \%$ (rango $32,0-84,0 \%$ ) en el resto.

La mayoría de los mortinatos anteparto están asociados con la restricción del crecimiento fetal (RCF); la RCF o los trastornos placentarios son más probables de ser detectados y el equilibrio de los riesgos podría favorecer la inducción temprana o la cesárea, aumentando la tasa de nacimientos prematuros (15). Sin embargo, la detección temprana de la RCF sigue siendo un reto incluso en los países desarrollados ${ }^{(16)}$.

A partir de 2001, por recomendaciones de expertos, la DIES registra una muerte fetal anteparto "si el feto presentaba señales de maceración" ${ }^{(7)}$. En un subanálisis y empleando esta definición la MF intraparto declinó de 47\% a $34 \%$ entre 2003 y 2013 (9\% sin información), reflejando mejoras en la atención obstétrica. 
Los resultados de este estudio sugieren que el aumento en las tasas de parto prematuro probablemente se deba más a modificaciones en las prácticas obstétricas que a alteraciones en las características demográficas de las embarazadas. Esto se vio ilustrado por la relación inversa y estadísticamente significativa entre el aumento de la tasa de nacimientos prematuros y el descenso de la mortalidad neonatal, explicable en parte por el progreso en la atención perinatal, especialmente en los prematuros extremos que aumentaron $36 \%$ entre 2011 y 2013.

Mientras que los elevados índices de parto prematuro y de parto muy prematuro siguen siendo indicadores de una deficiente salud perinatal de la población, las altas tasas de parto prematuro a fines de la gestación pueden ser indicativos de altas tasas de cesáreas sin la apropiada calidad de la atención neonatal. Esto se vio reflejado en la tasa de prematuros moderados, que presentó el mayor incremento entre 2011 y 2013 (0.75\%), representando el $87 \%$ del total de prematuros, mientras que el mayor descenso de la TMFE se observó entre 28 a 31 semanas $(68 \%)$ y postérminos $(29 \%)$. Por consiguiente, en los últimos años la tasa de nacimientos prematuros se ha convertido en una medida menos trascendente de la salud maternofetal-neonatal ${ }^{(6)}$.

En nuestro estudio describimos cambios seculares en la proporción de mujeres con características conocidas como factores de riesgo de muerte fetal y partos prematuros: mejoras de la baja escolaridad e incremento de embarazos múltiples, de adolescentes y mayores de 35 años. (Tabla 1) El aumento de la tasa de embarazadas de 35 años y más, y el crecimiento de la educación materna indican que las mujeres están posponiendo el inicio de su vida reproductiva, como en los países más desarrollados (17). Este cambio en el comportamiento reproductivo también se asoció con el aumento de nacimientos múltiples, que a su vez mostraron una disminución de la mortalidad fetal, pero asociado al incremento de nacimientos prematuros. (Tabla 2)

El aumento de los años de escolaridad materna (expresado como una disminución en la proporción con baja escolaridad) se asoció con disminución de la mortalidad fetal y aumento de los nacimientos prematuros. El aumento de nacimientos prematuros con mayor alfabetización femenina también se observó en un estudio ecológico de nacimientos prematuros de diferentes países ${ }^{(3)}$ y los autores sugirieron que era un reflejo del estilo de vida occidental que lleva a mejor acceso a la atención prenatal.
Una de las limitaciones del presente estudio es que se realizó con datos secundarios, basados en informes consolidados de estadísticas vitales nacionales y registros de nacimientos con un número limitado de variables de confusión, por lo que otras podrían estar presentes, pero no controladas. Aunque los datos disponibles no permiten la evaluación de la complejidad de la relación mortalidad fetal/parto prematuro, el análisis temporal de estos datos sugiere que la tendencia del crecimiento de los nacimientos prematuros podría atribuirse (parcialmente) por un lado al aumento de la interrupción del embarazo médicamente indicada, con el objetivo de reducir las pérdidas fetales, y por el otro al parto prematuro iatrogénico, sin indicación materna ni fetal. No fue posible estimar la contribución de los partos prematuros espontáneos o idiopáticos debido a no estar incluido en los consolidados. Solamente a partir de 2011 se contó con la EG de los RN vivos para calcular tendencias de las tasas de mortalidad fetal específicas por categorías de EG; sin embargo y dado el tamaño muestral de este período (190.319 prematuros) es muy probable que sea representativo $(29,4 \%$, IC 95\% 29,3 29,5) del total estudiado.

Las principales fortalezas fueron la fuente de datos que incluyó todas las muertes fetales y nacimientos vivos en un período reciente y la escasa proporción de datos incompletos, necesarios para estudiar de forma confiable tendencias de diferentes resultados y ajustarlos para potenciales factores de confusión.

\section{Conclusión}

La reducción de la mortalidad fetal se asoció con el incremento observado de los nacimientos vivos prematuros en la Argentina.

\section{Bibliografía}

1. Lawn J, Waiswa $P$, Amouzou A, Mathers $C$, Hogan $D$ et al. Stillbirths: rates, risk factors, and acceleration towards 2030. Lancet 2016; 387: 587-603.

2.Cousens S, Blencowe H, Stanton C, Chou D, Ahmed S et al. National, regional and worldwide estimates of stillbirth rates in 2009 with trends since 1995: a systematic analysis. Lancet 2011; 377:1319 - 30.

3.Blencowe H, Cousens S, Oestergaard M, Chou D, Moller $A$, et al. National, regional, and worldwide estimates of preterm birth rates in the year 2010 with time trends since 1990 for selected countries: a systematic analysis and implications. Lancet 2012; 379:2162-72.

4. Lisonkova S, Hutcheon J, Joseph K. Temporal trends in neonatal outcomes following iatrogenic preterm delivery. BMC Pregnancy Childbirth 2011; 25:39.

5. Alencar $G$, da Silva $Z$, Santos $P$, Raspantini $P$, et al. What is the impact of interventions that prevent fetal mortality on the increase of preterm live births in the 
State of Sao Paulo, Brazil? BMC Pregnancy and Childbirth 2015; 15:152.

6. Lisonkova S, Sabr Y, Butler B, Joseph K. International comparisons of preterm birth: higher rates of late preterm birth are associated with lower rates of stillbirth and neonatal death. BJOG 2012; 119:16309.

7. Estadísticas Vitales de la Dirección de Estadística e Información de Salud (DEIS), Ministerio de Salud, Argentina. [Disponible en: http://deis.msal. gov.ar/index.php/estadisticas-vitales/]. [Consulta: 21 de junio 2016].

8. World Health Organization. International statistical classification of diseases, and related health problems, 10th revision (ICD-10). Vol. 2. 2nd ed. Geneva (Switzerland); 1994

9. Millennium Development Indicators: World and regional groupings. [Disponible en: http://mdgs.un.org/unsd/mdg/Host.aspx?Content=Data/ RegionalGroupings] [Consulta: 12 de marzo 2016].

10. Stanton C, Lawn J, Rahman H, Wilczynska-Ketende K, Hill K. Stillbirth rates: delivering estimates in 190 countries. Lancet 2006; 367: 1487- 94.

11. Grandi C. Tasas de natalidad pretérmino crecientes, 1989-2004: ¿Se modifica la demografía o cambian las prácticas obstétricas? Arch Argent Pediatr 2012; 110: 268-277.

12. Passini R Jr, Cecatti J, Lajos G, Tedesco R, Nomura M, et al. Brazilian multicentre study on preterm birth (EMIP): prevalence and factors associated with spontaneous preterm birth. PLOS ONE 2014; 9: e109069.

13. Flenady V, Middleton $P$, Smith G. Stillbirths: the way forward in high-income countries. Lancet 2011; 377: $1703-17$.

14. Delnord M, Blondel B, Zeitlin J. What contributes to disparities in the preterm birth rate in European countries? Curr Opin Obstet Gynecol 2015; 27: 133-42.

15. de Bernis L, Kinney M, Stones W, Ten Hoope-Bender P, et al. Stillbirths: ending preventable deaths by 2030 . Lancet 2016; 387:703-16.

16. Flenady $V$, Wojcieszek $A$, Middleton $P$, Ellwood $D$, Erwich $\mathrm{J}$, et al, for The Lancet Ending Preventable Stillbirths study group and The Lancet Stillbirths in HighIncome Countries Investigator Group. Stillbirths: recall to action in high-income countries. Lancet 2016; 387: 691 702.

17. Lisonkova S, Paré E, Joseph K. Does advanced maternal age confer a survival advantage to infants born at early gestation? BMC Pregnancy \& Childbirth 2013; 13: 87. 